\title{
ELASTO-DYNAMIC INSTABILITY OF SHALLOW PIN-ENDED STEEL ARCHES UNDER CENTRAL STEP LOADING
}

\author{
Yong-Lin Pi ${ }^{1, *}$, Mark Andrew Bradford ${ }^{1}$ and Shuguo Liang ${ }^{2}$ \\ ${ }^{I}$ Centre for Infrastructure Engineering and Safety, School of Civil and Environmental Engineering \\ UNSW, Sydney, NSW 2052, Australia \\ ${ }^{2}$ School of Civil Engineering, Wuhan University, Wuhan 430071, P.R. China \\ *(Corresponding author: E-mail: y.pi@unsw.edu.au)
}

Received: 23 April 2008; Revised: 6 August 2008; Accepted: 11 August 2008

\begin{abstract}
When a shallow steel arch is subjected to in-plane central step loading, the arch will oscillate about an equilibrium position due to the kinetic energy imparted to the arch by the load. If the load is sufficiently large, the motion of the arch may reach an unstable equilibrium configuration that leads to elasto-dynamic instability of the arch. This paper presents a study of the in-plane elasto-dynamic instability of a shallow pin-ended circular arch under central step loading with infinite duration. The principle of conservation of energy is used to establish the criterion for elasto-dynamic instability, and analytical solutions for the lower and upper elasto-dynamic instability loads of the arch are obtained. It is found that the elasto-dynamic instability loads of a shallow pin-ended arch under central step loading with infinite duration are lower than its elasto-static counterparts.
\end{abstract}

Keywords: Bifurcation buckling, circular arch, dynamic instability, elastic, energy criterion, limit instability, step loading

\section{INTRODUCTION}

A shallow steel arch that is subjected to an in-plane central static load may buckle in the plane of loading in an elastic bifurcation mode or in an elastic limit instability mode when the load reaches a critical value. It has been found [1-5] that the prebuckling deformations have significant effects on the instability of a shallow arch and that classical buckling theory cannot predict the instability load of shallow arches correctly. Analytical solutions for limit instability and bifurcation buckling loads for shallow arches under a central load have been obtained [1]. When the central load is suddenly applied to the arch at time $t=0$ as a step load (Figure 1), the load will impart kinetic energy to the arch, and dynamic effects of the step loading on the instability of the arch have to be considered. When the step load is sufficiently small, it causes the arch to oscillate about a stable equilibrium position and the amplitudes of oscillation are sufficiently small. If this load is sufficiently large, the motion of the arch may reach an unstable equilibrium position, which may then induce elasto-dynamic buckling of the arch.

Commonly, the equations of motion of an arch are solved numerically for various values of the load parameter to obtain the response of the system [6-12]. The load parameter at which there exists a large increase of the transient response is considered as the critical one [6]. The major merit of this method is that it considers the dynamic effects directly and intuitively. However, when this method is applied to a continuum such as a shallow arch, a number of approximations have to be made. Power series expansion of the displacement components are usually used to obtain approximate solutions for the elasto-dynamic instability [13-14]. For convenience in obtaining dynamic solutions, the effects of the transverse deformation on the axial deformation are usually ignored [6-14]. However, the effects of transverse (normal) deformations on axial deformations in a circular shallow arch are significant and so should be considered, particularly when exact closed form solutions are sought, as was pointed out in [1-5]. In addition, most investigations of the dynamic buckling of shallow arches using this method have focused on sinusoidal arches under loads distributed as half sine-waves $[6,7,9,10]$. 


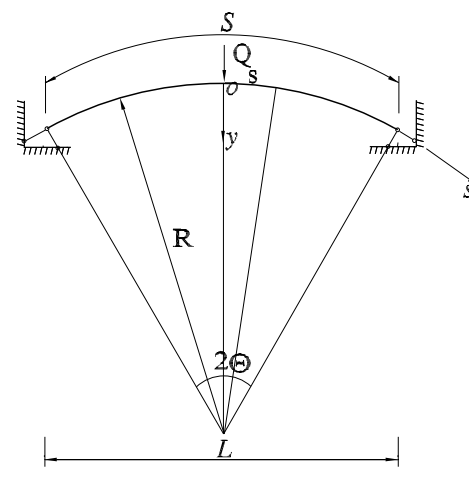

(a) Pin-ended arch

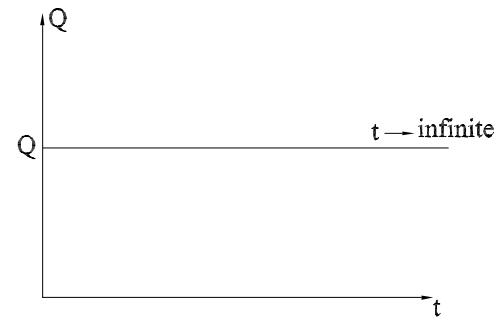

(b) Step loading with infinite duration

Figure 1. Arch and Load

The total energy phase plane of a structural system is also used to predict the elasto-dynamic instability of arches $[8,9,11,12]$. The motion of the arch can be represented by a trajectory in a functional phase space which can be taken as a Euclidean space of infinite dimensions. Critical conditions are related to the characteristics of the system's phase plane or space, and the emphasis is on establishing sufficient conditions for stability and for instability [6-9].

The principle of conservation of energy $[9,15-17]$ can be applied to the elasto-dynamic instability analysis of a conservative system. The major merit of the method is that it is devoted to finding the criterion which allows the elasto-dynamic buckling load to be determined without actually having to solve the equations of motion per se.

In this paper, attention will be directed to using the principle of energy conservation to investigate the elasto-dynamic buckling of shallow pin-ended circular steel arches that are subjected to a central step loading of infinite duration as shown in Figure 1.

\section{ENERGY CRITERION FOR ELASTO-DYNAMIC INSTABILITY}

\subsection{Equation of Motion Approach}

In this section, a one degree-of-freedom idealized arch system shown in Figure 2 is used to establish the energy criterion for dynamic instability. The system consists of two rigid bars with the same length $L$ pinned together and the other ends of the two bars are pin-ended or simply supported (Figure 2). A mass $m$ is attached to the pin-joint and a linear translational spring is connected to the pin-joint with the dimensions as shown in Figure 2. The stiffness of the spring is $k$. The system is assumed to have an initial angle $\theta_{0}$. The motion of the system under a sudden horizontal load $Q$ can be described by the rotation angle $\theta$.

Before developing the energy criterion for dynamic instability, the equation of motion approach is used to investigate the dynamic instability of the one degree-of-freedom arch system. The equation of motion for the system can be obtained in a dimensionless form as

$$
\ddot{\theta}(\tau)+\left(1-\frac{\sqrt{1+\sin \theta(0)}}{\sqrt{1+\sin \theta(\tau)}}\right) \cos \theta(\tau)-\bar{Q} \sin \theta(\tau)=0,
$$


where $\ddot{\theta}=\partial^{2} \theta / \partial \tau^{2}$ is the dimensionless angular acceleration of the system, and the dimensionless time parameter $\tau$ is defined by $\tau=t \sqrt{k / m}$, and the dimensionless load $\bar{Q}$ is defined by $\bar{Q}=2 Q / k L \quad$ (Figure 2), in which $t$ is the time.

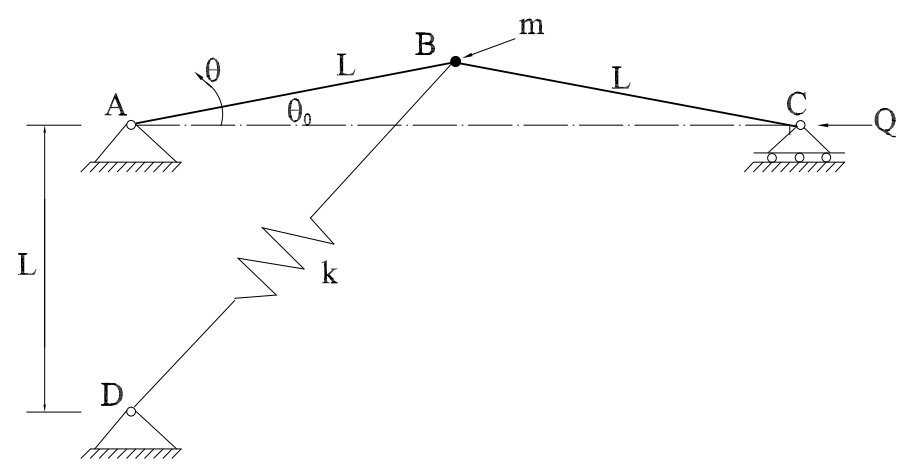

Figure 2. One Degree-of-Freedom System

The initial value problem given by Eq. 1 can be solved by numerical schemes such as the Runge-Kutta procedure when the initial conditions at $\tau=0$ are given. For example, when the initial rotation angle $\theta(0)=0.008$ and the initial angular velocity $\dot{\theta}(0)=0$ at $\tau=0$ are assumed, the results that are obtained for the dimensionless load $\bar{Q}=0.4000,0.4100,0.4153$, and 0.4154 are shown in Figure 3 as variations of the rotational angular response $\theta(\tau)$ with the dimensionless time $\tau$. It can be seen from Figure 3 that when the load $\bar{Q}=0.4000,0.4100$, or 0.4153 is suddenly applied, the motion of the system is simply oscillatory with small amplitudes. The oscillation takes place between the initial position $\theta(0)=0.008$ and a maximum amplitude of $\theta(\tau)$. When the load $\bar{Q}=0.4154$ is suddenly applied, the amplitude of the motion of the system becomes so large that an escaping motion, i.e. dynamic instability of the system, occurs as shown in Figure 3.

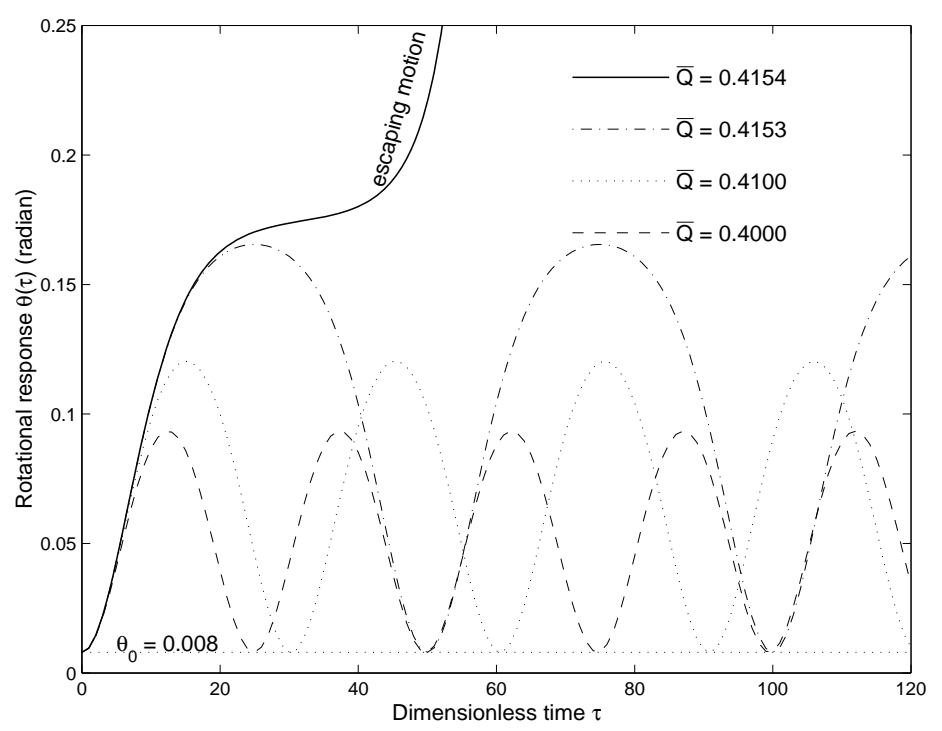

Figure 3. Variations of the Rotational Response with Dimensionless Time 
Variations of the maximum response amplitude $\theta_{\max }$ with the dimensionless load $\bar{Q}$ are shown in Figure 4. It can be seen that there is large jump in the maximum amplitude $\theta_{\max }$ at $\bar{Q}=0.4154$. According to the Budiansky-Roth criterion [6], the dynamic buckling load is estimated to be $\bar{Q}=$ 0.4154 .

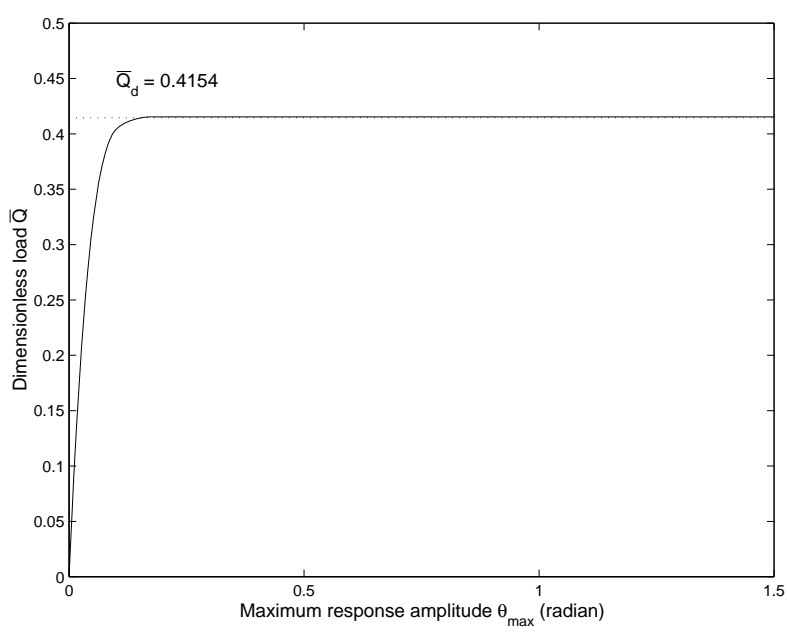

Figure 4. Variations of the Maximum Amplitude $\theta_{\max }$ with the Dimensionless Load $\bar{Q}$

\subsection{Energy Criterion for Elasto-Dynamic Instability}

Although the equation of motion approach can be used with numerical methods to deal with a continuum subjected to a number of initial conditions, calculations in this approach require an excessive amount of time, which often makes its application very difficult. In addition, the accuracy of this approach often depends on the number of degrees-of-freedom of the reduced system and on the accuracy of the numerical method adopted [13-14].

For a dynamic instability analysis, what is actually needed is to establish the critical states for the instability and so the complete dynamic solution is usually not needed. Herein, an effort will be devoted to finding the criterion that makes it possible to determine the dynamic instability load without actually having to solve the equations of motion for the arch system. A dynamic load generally is one whose magnitude, direction, or point of application varies with time, and it is difficult to solve the dynamic instability problem of a structure analytically under a general dynamic load. This paper concentrates only on step loading with infinite duration, which is a special form of dynamic loading and is suddenly applied at the time $t=0$.

Because the structure and the step load of infinite duration form a conservative system, the total energy of the system must satisfy the principle of energy conservation, i.e.

$\bar{E}(\dot{\theta}, \theta)=\bar{T}(\dot{\theta})+\bar{U}(\theta)=$ constant

where $\bar{E}(\dot{\theta}, \theta)$ is the dimensionless total energy, $\bar{T}(\dot{\theta})$ is the dimensionless kinetic energy of the system and is given by

$\bar{T}(\dot{\theta})=\frac{m[L \dot{\theta}(t)]^{2}}{2 k L^{2}}=\frac{1}{2}[\dot{\theta}(\tau)]^{2}$ 
and $\bar{U}(\theta)$ is the dimensionless total potential energy of the system and is given by

$\bar{U}(\theta)=\left(\sqrt{1+\sin \theta}-\sqrt{1+\sin \theta_{0}}\right)^{2}-\bar{Q}\left(\cos \theta_{0}-\cos \theta\right)$.

The necessary conditions for extremes of the total energy $\bar{E}(\dot{\theta}, \theta)$ are given by

$\partial \bar{E}(\dot{\theta}, \theta) / \partial \dot{\theta}=\partial \bar{T}(\dot{\theta}) / \partial \dot{\theta}=0$

and

$\partial \bar{E}(\dot{\theta}, \theta) / \partial \theta=\partial \bar{U}(\theta) / \partial \theta=0$

The condition given by Eq. 5 leads to

$\dot{\theta}(\tau)=0$

The value of the total energy $\bar{E}(\dot{\theta}, \theta)$ of the system is determined by the initial condition before it is subjected the step loading. At $t=0$, it is assumed that the system is free from loading and is at rest, and so $\dot{\theta}(0)=0$ and the displacement $\theta(0)=\theta_{0}$. In this case, the total energy $\bar{E}(\dot{\theta}(0), \theta(0))=0$ at $t=0$. Because of the principle of energy conservation, for $t>0$

$\bar{E}(\dot{\theta}, \theta)=\bar{T}(\dot{\theta})+\bar{U}(\theta)=\bar{E}(\dot{\theta}(0), \theta(0))=0$.

Substituting Eqs. 3 and 7 into Eq. 8 leads to

$\bar{U}(\theta)=0$,

which indicates that vanishing of the total potential energy is a necessary condition for extremes of the total energy.

Eq. 6 leads to another necessary condition for extremes of the total energy as

$\bar{Q}=\frac{\left(\sqrt{1+\sin \theta}-\sqrt{1+\sin \theta_{0}}\right) \cos \theta}{\sin \theta \sqrt{1+\sin \theta}}$

which is also the condition for the static equilibrium configurations of the system.

Variations of the total potential energy $\bar{U}(\theta)$ with rotation angle $\left(\theta-\theta_{0}\right)$ are shown in Figure 5, where $A_{i}$ and $B_{i}(i=1,2,3,4)$ are the equilibrium positions obtained by setting $\partial \bar{U} / \partial \theta=0$. Because the kinetic energy $\bar{T}(\dot{\theta})$ of the system is a positive definite function of the velocity $\dot{\theta}$ and the system is at rest at $t=0$ prior to loading, motion of the system is possible when the total potential energy $\bar{U}(\theta)$ is non-positive and so the principle of energy conservation given by Eq. 8 
holds. It can be seen from Figure 5 that for loads $\bar{Q}=0.4000$ or 0.4100 , the total potential energy $\bar{U}(\theta)<0$ at a stable equilibrium position $A_{i}$, so the principle of energy conservation given by Eq. 8 holds and the system will oscillate about $A_{i}$. However, the total potential energy of the system $\bar{U}(\theta)>0$ at the unstable equilibrium position $B_{i}$, so the principle of energy conservation given by Eq. 8 is violated and the oscillation of the system cannot reach the unstable equilibrium position $B_{i}$. When the total potential energy at an unstable equilibrium position vanishes $(\bar{U}(\theta)=0)$, the principle of energy conservation given by Eq. 8 holds if the kinetic energy also vanishes. In this case, the oscillation of the system can reach the unstable equilibrium position and so dynamic buckling is possible. The dynamic buckling load and the corresponding rotation angle can then be obtained by solving $\bar{U}(\theta)=0$ given by Eq. 9 and $\partial \bar{U}(\theta) / \partial \theta=0$ given by Eq. 10 simultaneously as $\bar{Q}_{d}=0.4154$ and $\theta=0.174698$ ( $B_{3}$ in Figure 5), which is the same as that obtained from the equation of motion.

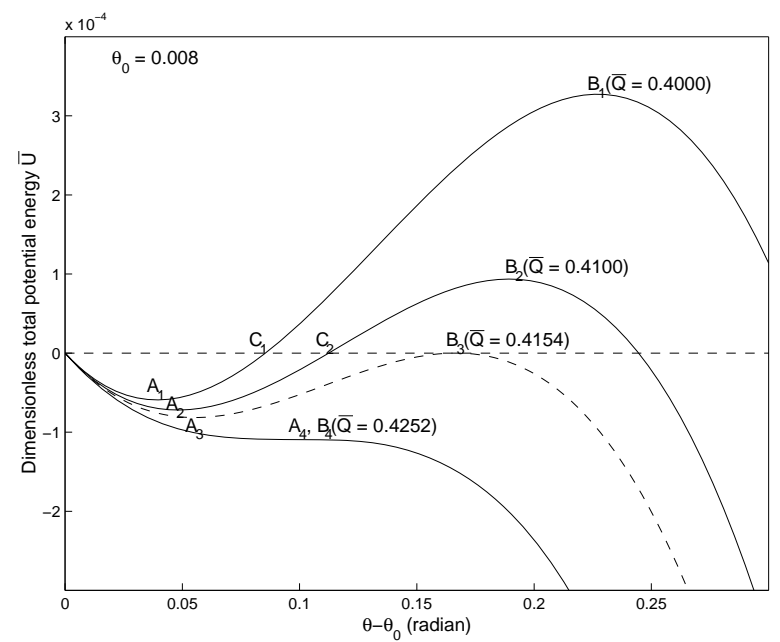

Figure 5. Variation of Energy with Rotation
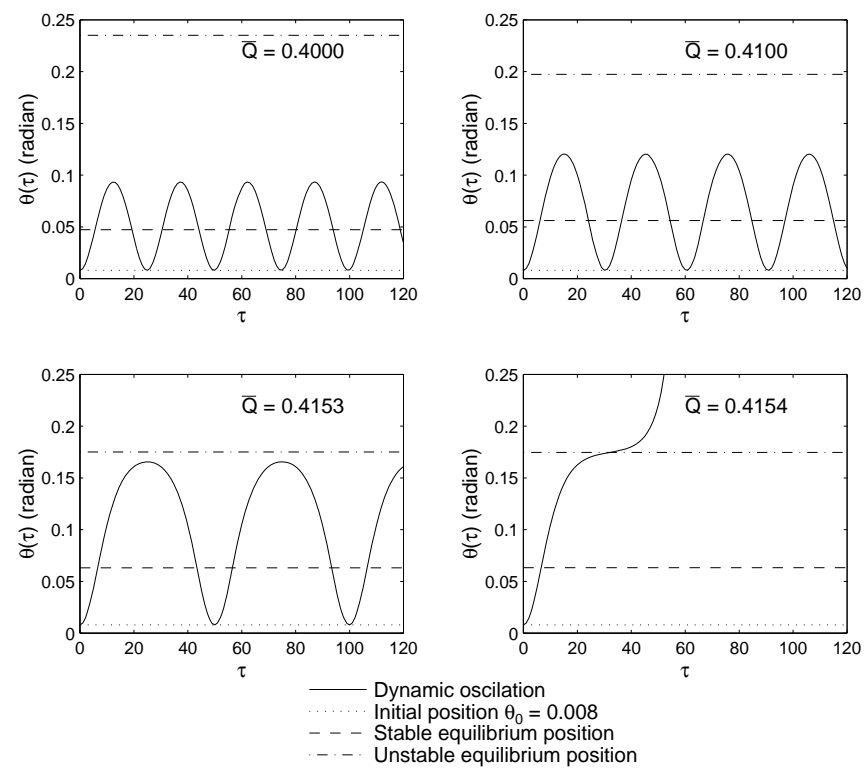

Figure 6. Dynamic Responses and Static Equilibrium 
The static equilibrium positions of the arch system given by Eq. (10) are compared with its dynamic responses given by Eq. 1 in Figure 6 for loads $\bar{Q}=0.4000,0.4100,0.4153$, and 0.4154 . It can be seen that when the load $\bar{Q}=0.4000,0.4100$ and 0.4153 , the arch system oscillates through the stable equilibrium positions $\theta=0.047307,0.056118$, and 0.063097 respectively and that the oscillations cannot reach the unstable equilibrium positions $\theta=0.235087$ (for $\bar{Q}=0.4000$ ), 0.197357 (for $\bar{Q}=0.4100$ ), and 0.175143 (for $\bar{Q}=0.4153$ ). However, when the load $\bar{Q}=0.4154$, the arch system moves through the stable equilibrium position $\theta=0.06326$ first, and then reaches the unstable equilibrium position $\theta=0.174698$ and escaping motion, i.e. dynamic instability of the system occurs.

If the load is slowly applied to the system, it can be considered as a static load and the system may buckle in a static instability mode. The static instability load of the system needs to satisfy

$$
\frac{\partial^{2} \bar{U}}{\partial \theta^{2}}=0
$$

Solving Eqs. 10 and 11 simultaneously yields the static instability load as $\bar{Q}_{s}=0.4252$ (Figure 5) which is higher than the dynamic instability load $\bar{Q}_{d}=0.4154$.

\section{DIFFERENTIAL EQUATIONS OF MOTION FOR SHALLOW STEEL ARCHES}

It is assumed that the dynamic response of the arch is undamped and so the Lagrangian $\mathcal{L}$ of the shallow pin-ended steel arch and load system can be expressed as

$$
\mathcal{L}=T-U
$$

where $T$ is the kinetic energy of the system given by

$$
T=\frac{1}{2} \int_{V} m\left(\dot{v}^{2}+\dot{w}^{2}\right) \mathrm{d} V=\frac{m A}{2} \int_{-S / 2}^{S / 2}\left(\dot{v}^{2}+\dot{w}^{2}\right) \mathrm{ds}
$$

and $U$ is the total potential energy of the system given by

$$
\begin{aligned}
& U=\frac{1}{2} \int_{-S / 2}^{S / 2} \int_{A} E \varepsilon^{2} \mathrm{~d} A \mathrm{~d} s-\int_{-S / 2}^{S / 2} \delta(s) Q v \mathrm{~d} s \\
& =\int_{-S / 2}^{S / 2}\left[\frac{1}{2}\left(A E \varepsilon_{m}^{2}+E I_{x} v^{\prime \prime 2}\right)-\delta(s) Q v\right] \mathrm{d} s,
\end{aligned}
$$

where $\bar{\delta}(s)$ is the Dirac-delta function, $E$ is the Young's modulus of steel, $A$ is the area of the cross-section, and $I_{x}$ is the second moment of area of the cross-section about its major principal axis.

In Eq. 14, the longitudinal normal strain $\varepsilon$ at an arbitrary point of the cross-section consists of the membrane strain $\varepsilon_{m}$ and bending strain $\varepsilon_{b}$ as $[1,2]$

$$
\varepsilon=\varepsilon_{m}+\varepsilon_{b} \quad \text { with } \quad \varepsilon_{m}=w^{\prime}-v / R+v^{\prime 2} / 2 \quad \text { and } \quad \varepsilon_{b}=-y v^{\prime \prime} \text {, }
$$


where ()$^{\prime}=\partial() / \partial s, s$ is the axial coordinate (Figure 1), $v$ and $w$ are the radial and axial displacements of the centroid, $R$ is the initial radius of the circular arch, and $y$ is the coordinate of the arbitrary point in the principal axis system of the cross-section.

The equations of motion can be derived from Hamilton's principle which can be stated as

$\int_{t_{1}}^{t_{2}} \delta \mathcal{L} \mathrm{d} t=\int_{t_{1}}^{t_{2}} \delta(T-U) \mathrm{d} t=0$ with $\delta v=\delta w=0$ at $t=t_{1}, t_{2}$ for $-S / 2 \leq s \leq S / 2$

Integrating Eq. 16 by parts and considering that $\delta v$ and $\delta w$ are arbitrary, leads to the differential equations of motion as

$\varepsilon_{m}^{\prime}+m A \ddot{w}=0 \quad$ and $\quad E I_{x} v^{i v}-A E \varepsilon_{m} v^{\prime \prime}-A E \varepsilon_{m} / R+m A \ddot{v}=\bar{\delta}(s) Q / R$,

in the axial and radial directions; and to the boundary conditions $v=v^{\prime \prime}=w=0$ at $s= \pm S / 2$.

In this study, the arch is assumed to be free from loading and at rest at $t=0$ before the step loading. Hence, the initial conditions of the arch are $v=\dot{v}=\ddot{v}=w=\dot{w}=\ddot{w}=0 \quad$ at $t=0$ for $-S / 2 \leq s \leq S / 2$.

\section{ELASTO-STATIC INSTABILITY OF SHALLOW STEEL ARCHES}

\subsection{Nonlinear Elasto-Static Equilibrium}

In order to use the principle of energy conservation to investigate the dynamic instability of a shallow arch under central step loading of infinite duration, knowledge of the static primary equilibrium path and of the static instability of the arch under a central static load is essential. The static instability of a shallow pin-ended circular arch was investigated by Bradford et al. [1]. For the static instability analysis, the displacements $v$ and $w$ are independent of time $t$ and so $\dot{v}=\ddot{v}=\dot{w}=\ddot{w}=0$.

From the first equation of Eq. 17, the membrane strain is a constant and can be written as

$\varepsilon_{m}=-\frac{N}{A E}$

where $N$ is the axial compressive force.

The differential equation of equilibrium for the radial direction can then be obtained from the second differential equation of motion of Eq. 17 as

$\frac{v^{i v}}{\mu^{2}}+v^{\prime \prime}=\frac{\bar{\delta}(s) Q}{N R}-\frac{1}{R}$,

where $\mu$ is the axial force parameter and is defined by

$\mu^{2}=\frac{N}{E I_{x}}$ 
The solution for $v$ can be obtained from Eq. 19 and the boundary conditions $v=v^{\prime \prime}=0$ at $s= \pm S / 2$ as

$$
\begin{aligned}
& v=\frac{1}{\mu^{2} R}\left\{1-\frac{\cos (\mu s)}{\cos (\mu S / 2)}+\frac{1}{2}\left[(\mu S / 2)^{2}-(\mu s)^{2}\right]\right. \\
& \left.+\frac{\bar{Q}}{\mu S / 2}[\tan (\mu S / 2) \cos (\mu s)-\mu S / 2+H(s)(\mu s-\sin (\mu s))]\right\}
\end{aligned}
$$

where the dimensionless load $\bar{Q}$ is given by

$$
\bar{Q}=\frac{Q(S / 2)^{2}}{2 \Theta E I_{x}}=\frac{Q R S}{4 E I_{x}}
$$

and the step function $H(\theta)$ is given by

$$
H(s)=\left\{\begin{array}{cc}
1 & \text { for } s>0 \\
-1 & \text { for } s<0
\end{array}\right. \text {. }
$$

From Eqs. 15 and 18, it can be obtained that

$$
w^{\prime}=-\frac{N}{A E}+\frac{v}{R}-\frac{v^{\prime 2}}{2} \text {. }
$$

Substituting Eq. 21 into Eq. 24 and integrating it over the entire arch leads to the nonlinear equation of equilibrium for the external central load and the internal axial force as

$$
A_{1} \bar{Q}^{2}+B_{1} \bar{Q}+C_{1}=0
$$

where

$$
\begin{aligned}
& A_{1}=\frac{1}{4(\mu S / 2)^{4}}\left[\tan ^{2}(\mu S / 2)-\frac{3 \tan (\mu S / 2)}{\mu S / 2}+3\right], \\
& B_{1}=\frac{1}{2(\mu S / 2)^{4}}\left[\frac{2 \cos (\mu S / 2)-\mu S / 2 \sin (\mu S / 2)}{\cos ^{2}(\mu S / 2)}-2\right], \\
& C_{1}=\left(\frac{\mu S}{2 \lambda}\right)^{2}+\frac{\mu S / 2-\sin (\mu S / 2) \cos (\mu S / 2)}{4(\mu S / 2)^{3} \cos ^{2}(\mu S / 2)}-\frac{1}{6} \text { with } \lambda=\frac{\Theta}{2} \frac{S / 2}{r_{x}}=\frac{S^{2}}{4 r_{x} R},
\end{aligned}
$$

in which $\lambda$ is the geometric parameter of the arch, which is a function of the half included angle $\Theta$ and the slenderness $S / r_{x}$ of the arch.

\subsection{Limit Instability}

A typical nonlinear elastic behaviour obtained from Eqs. 21 and 25 for an arch with a geometric parameter $\lambda=6.5$ is shown in Figure $7 \mathrm{a}$ as variations of the dimensionless central displacement 
$v_{c} / f$ with the dimensionless load $\bar{Q}$, and in Figure $7 \mathrm{~b}$ as variations of the internal axial force parameter $\mu S / 2$ with the dimensionless load $\bar{Q}$, where $v_{c}$ is the central radial displacement and $f$ is the arch rise. It is assumed that the arch is loaded in a displacement-controlled manner. It can be seen from Figures $7 \mathrm{a}$ and $7 \mathrm{~b}$ that as the displacement increases, the load and the internal force parameter increase along the stable equilibrium path $0 a$ until the upper limit point $a$ is reached. As the displacement continues to increase, the load decreases along the unstable equilibrium path $a b$ and the internal axial force parameter increases first and then decreases until the lower limit point $b$ is reached. As the displacement increases further, the load increases again but the internal axial force continues to decrease along the stable equilibrium path $b f$. The loads corresponding to the upper and lower limit points are known as the upper and lower limit instability loads, respectively.

However, in practice, an arch is usually loaded in a load-controlled manner. When the load is increased further from the limit point $a$ by an infinitesimal amount, there is no adjacent stable equilibrium state and the only possible stable equilibrium state exists at a finite distance apart, i.e. at the state corresponding to the stable equilibrium point $c$ as shown in Figures $7 \mathrm{a}$ and $7 \mathrm{~b}$. Therefore, the arch snaps through from the limit point $a$ to the equilibrium point $c$, as indicated by the dashed lines in Figures $7 \mathrm{a}$ and $7 \mathrm{~b}$, which are not an equilibrium path.

When the external load decreases, the arch follows the path $f_{c} b$ until the lower limit point $b$ is reached. If the load is further decreased by an infinitesimal amount, there is no adjacent stable equilibrium state and the arch will snap-through to the stable equilibrium point $d$. Because of the snap-through phenomenon, the upper and lower limit instability loads are also known as the upper and lower snap-through buckling loads.

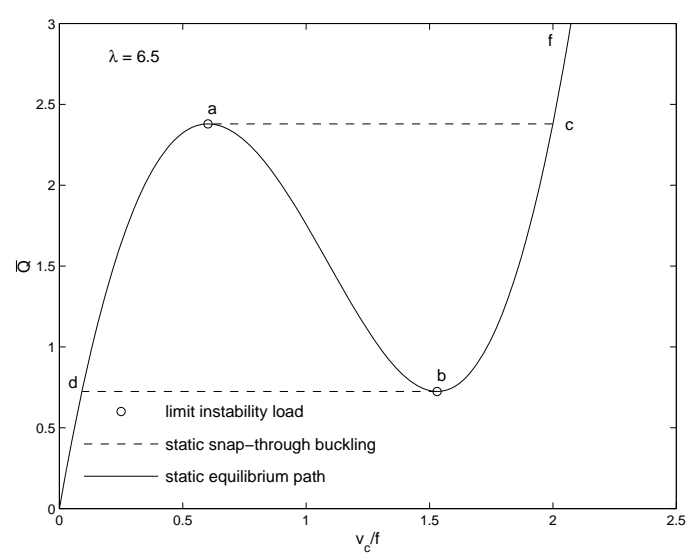

a. Variations of Dimensionless Central Displacement with Dimensionless Load

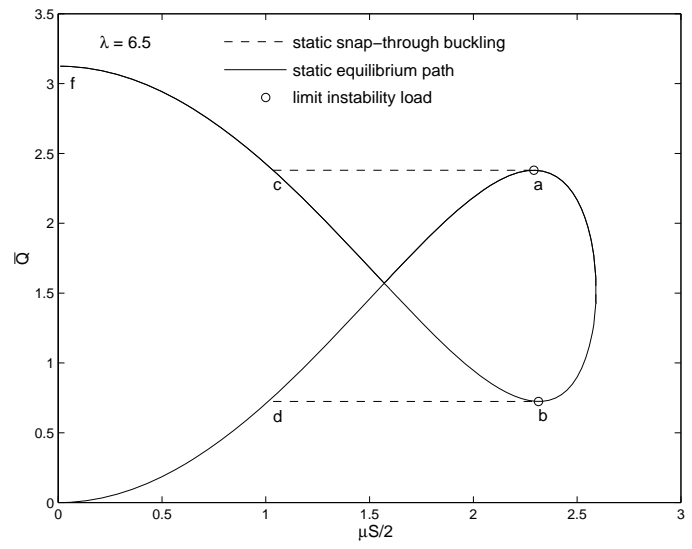

b. Variations of Dimensionless Axial Force with Dimensionless Load

Figure 7. Static Limit Instability

Eq. 25 expresses the load $\bar{Q}$ as an implicit function of the axial force parameter $\mu$ as $F(\bar{Q}, \mu)=0$. Setting $\partial \bar{Q} / \partial \mu=-(\partial F(\bar{Q}, \mu) / \partial \mu) /(\partial F(\bar{Q}, \mu) / \partial \bar{Q})$ leads to the following equation for the upper and lower limit points $a$ and $b$

$A_{2} \bar{Q}^{2}+B_{2} \bar{Q}+C_{2}=0$,

$A_{2}=\frac{1}{8(\mu S / 2)^{4}}\left[8-\frac{15 \tan (\mu S / 2)}{8(\mu S / 2)}+\frac{7}{\cos ^{2}(\mu S / 2)}-\frac{2(\mu S / 2) \tan (\mu S / 2)}{\cos ^{2}(\mu S / 2)}\right]$, 


$$
\begin{aligned}
& B_{2}=\frac{1}{4(\mu S / 2)^{4}}\left[\frac{8}{\cos (\mu S / 2)}-8-\frac{5(\mu S / 2) \tan (\mu S / 2)}{\cos (\mu S / 2)}-\frac{(\mu S / 2)^{2}}{\cos (\mu S / 2)}+\frac{2(\mu S / 2)^{2}}{\cos (\mu S / 2)}\right], \\
& C_{2}=\frac{1}{8(\mu S / 2)^{2}}\left[\frac{3}{\cos ^{2}(\mu S / 2)}-\frac{3 \tan (\mu S / 2)}{\mu S / 2}-\frac{2(\mu S / 2) \tan (\mu S / 2)}{\cos ^{2}(\mu S / 2)}\right]-\frac{(\mu S / 2)^{2}}{\lambda^{2}} .
\end{aligned}
$$

The limit instability loads obtained by solving Eqs. (25) and (29) simultaneously are shown in Figures $7 \mathrm{a}$ and $7 \mathrm{~b}$.

\subsection{Bifurcation Buckling}

Considering equilibrium in the prebuckled and the adjacent buckled configurations, and that the buckling membrane strain $\varepsilon_{m b}=0$, leads to the differential equation for elasto-bifurcation buckling of the arch as

$$
\frac{v_{b}^{i v}}{\mu^{2}}+v_{b}^{\prime \prime}=0
$$

where $v_{b}$ is the buckling displacement. The solution of Eq. 33 can be written as

$$
v_{b}=E_{1} \cos (\mu s)+E_{2} \sin (\mu s)+E_{3} s+E_{4} .
$$

Substituting Eq. 34 into the boundary conditions $v_{b}=v_{b}^{\prime \prime}=0$ at $s= \pm S / 2$ leads to a group of four homogeneous algebraic equations for $E_{1}, E_{2}, E_{3}, E_{4}$. The condition for nontrivial solutions of the group of equations for $E_{1}, E_{2}, E_{3}$, and $E_{4}$ leads to a characteristic equation as

$$
\sin (\mu S / 2) \cos (\mu S / 2)=0 .
$$

The fundamental solution of the first factor $\sin (\mu S / 2)=0$ of Eq. 35 is $\mu S / 2=\pi$ and substituting this into the nonlinear equation of equilibrium given by Eq. 25 yields the equation for the bifurcation buckling load as

$$
A_{3} \bar{Q}^{2}+B_{3} \bar{Q}+C_{3}=0 \quad \text { with } \quad A_{3}=\frac{3}{4 \pi^{4}}, \quad B_{3}=-\frac{2}{\pi^{4}}, C_{3}=\frac{1}{4 \pi^{2}}-\frac{1}{6}+\frac{\pi^{2}}{\lambda^{2}}
$$

Typical bifurcation buckling behaviour for an arch with a geometric parameter $\lambda=13$ obtained from Eqs. 22, 25 and 36 is shown in Figure 8a as variations of the dimensionless central displacement $v_{c} / f$ with the dimensionless load $\bar{Q}$ and in Figure $8 \mathrm{~b}$ as variations of the internal axial force parameter $\mu S / 2$ with the dimensionless load $\bar{Q}$. It can be seen from Figures 8 a and $8 \mathrm{~b}$ that as the displacement increases, the load increases along the stable equilibrium path $0 a$ until the upper bifurcation point $a$ is reached. As the displacement continues to increase, the internal axial force remains a constant but the load decreases along the secondary bifurcation unstable equilibrium path $a b$ until the lower bifurcation point $b$ is reached and at the lower bifurcation point $b$, equilibrium of the arch bifurcates from the secondary bifurcation equilibrium path back to the primary stable equilibrium path $b f$. After that, as the displacement increases, the load again 
increases but the internal axial force continues to decrease along the stable equilibrium path $b f$. The curve for the primary equilibrium path is also shown in Figures $8 \mathrm{a}$ and $8 \mathrm{~b}$. It can be seen that the upper limit instability load at the limit point $a_{s}$ is higher than the upper bifurcation buckling load, while the lower limit instability load at the limit point $b_{s}$ is lower than the lower bifurcation buckling load. Hence, limit instability of this arch cannot occur.

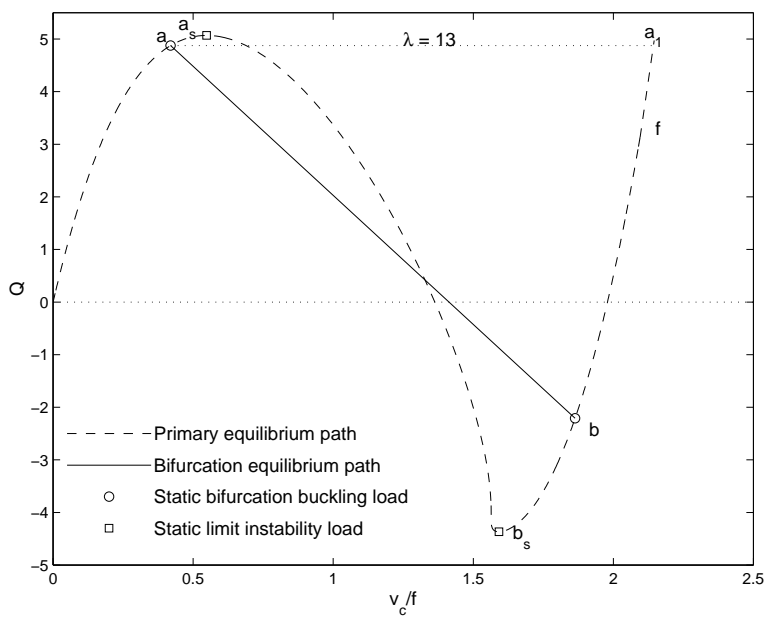

a. Variations of Dimensionless Central Displacement with Dimensionless Load

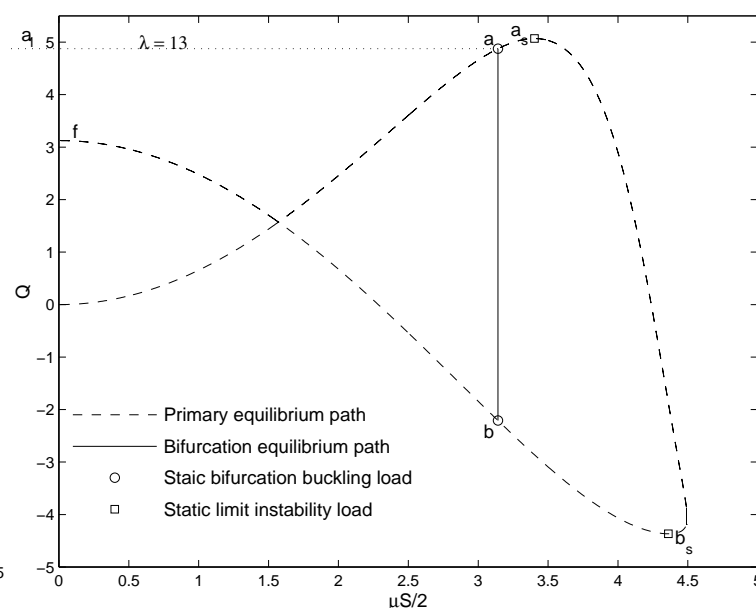

b. Variations of Dimensionless Axial Force with Dimensionless Load

Figure 8. Bifurcation Buckling

In practice, an arch is usually loaded in a load-controlled manner. When the load is increased further from the bifurcation buckling point $a$ by an infinitesimal amount, there is no adjacent stable equilibrium state and the only possible equilibrium state exists at a finite distance apart, i.e. at the state corresponding to a remote stable equilibrium point $a_{1}$ as shown in Figures 8a and 8b. Similarly to the limit instability, the arch snaps from the bifurcation buckling point $a$ to the remote stable equilibrium point $a_{1}$, as indicated by the dashed lines in Figures 8a and 8b, which is not an equilibrium path.

\section{ELASTO-DYNAMIC ISTABILITY OF SHALLOW STEEL ARCHES}

The criterion for the elasto-dynamic instability developed for the one degree-of-freedom idealized arch system can be applied to the elasto-dynamic instability analysis of shallow pin-ended arches under central step loading with infinite duration.

The dimensionless total potential energy of the arch and load system can be obtained by substituting solutions for the membrane strain $\varepsilon_{m}$ given by Eq. 18 and for the radial displacement $v$ given by Eq. 21 into the expression for the dimensionless total potential energy given by Eq. 14 and then integrating the expression as

$U=\frac{E I_{x}}{R^{2}}\left(A_{4} \bar{Q}^{2}+B_{4} \bar{Q}+C_{4}\right)$,

where 


$$
\begin{aligned}
& A_{4}=\frac{\left[(\mu S / 2)+4(\mu S / 2) \cos ^{2}(\mu S / 2)-5 \sin (\mu S / 2) \cos (\mu S / 2)\right]}{2 \mu^{3}(S / 2)^{2} \cos ^{2}(\mu S / 2)}, \\
& B_{4}=\frac{\left\{4 \cos (\mu S / 2)-\left[4+(\mu S / 2)^{2}\right] \cos ^{2}(\mu S / 2)-\mu S / 2 \sin (\mu S / 2)\right\}}{\mu^{2} S / 2 \cos ^{2}(\mu S / 2)}, \\
& C_{4}=\frac{S}{2}+\frac{S}{4 \cos ^{2}(\mu S / 2)}-\frac{3 \tan (\mu S / 2)}{2 \mu}+\frac{\mu^{2} S^{3}}{8}\left(\frac{\mu S / 2}{\lambda}\right)^{2} .
\end{aligned}
$$

Before being subjected to the central step loading, the shallow arch is assumed to be free from loading and at rest. Hence, the total energy of the arch is equal to zero, i.e. $E=0$ at $t=0$. From the principle of energy conservation, for $t>0$

$$
E=T+U=0 .
$$

It is noted that the kinetic energy $T$ of the arch imparted by the step loading is a positive definite function of the velocities. When the load is sufficiently small, from Eq. 14, the potential energy $U$ of the arch and load system at a stable equilibrium position is non-positive, the principle of energy conservation given by Eq. 41 may hold and the arch will oscillate about the stable equilibrium position. However, when the load is so small that the total potential energy $U$ of the system at an unstable equilibrium position given by Eq. 14 is positive, the total energy $E$ does not vanish and the principle of energy conservation given by Eq. 41 is violated. In this case, the motion of the arch to the unstable equilibrium position is impossible. As the value of the step load increases, it can be obtained from Eq. 14 that the total potential energy $U$ decreases. Because the kinetic energy of the arch is a positive definite function, its minimum value is equal to zero. From the principle of energy conservation given by Eq. 41, the necessary condition that the arch can move to the unstable equilibrium position with the zero kinetic energy is vanishing of the total potential energy $U$ of the arch, i.e.

$$
U=A_{4} \bar{Q}^{2}+B_{4} \bar{Q}+C_{4}=0 .
$$

In this case, the elasto-dynamic instability of the arch under the central step loading of infinite duration is possible. Because the elasto-dynamic instability load corresponds to an unstable equilibrium position, it also needs to satisfy the equilibrium equation given by Eq. 25 . The elasto-dynamic instability load can then be obtained by solving Eqs. 25 and 42 simultaneously. Since Eq. 25 describes the primary equilibrium of the arch, the solution obtained is the upper elasto-dynamic instability load.

The total potential energy may also vanish on the secondary bifurcation unstable equilibrium path. In this case, the lower elasto-dynamic instability load can be obtained by solving Eq. 42 with $\mu S / 2=\pi$.

Typical solutions for the upper and lower elasto-dynamic instability loads for an arch with an arch geometric parameter $\lambda=14$ are shown in Figure 9, where the solid line is the primary equilibrium path under static loading, the dashed line is the secondary equilibrium path for bifurcation buckling, and the dot-dashed line represents the variation of the dimensionless load $\bar{Q}$ and axial force parameter $\mu S / 2$ for the condition of zero total potential energy $(U=0)$. The intersection point $d_{u}$ of the zero total potential energy curve and the unstable branch $a_{s} b_{s}$ of the primary equilibrium path 
defines the upper elasto-dynamic instability load while the intersection point $d_{l}$ on the zero total potential energy curve and the secondary bifurcation equilibrium path $a b$ defines the lower elasto-dynamic instability load.

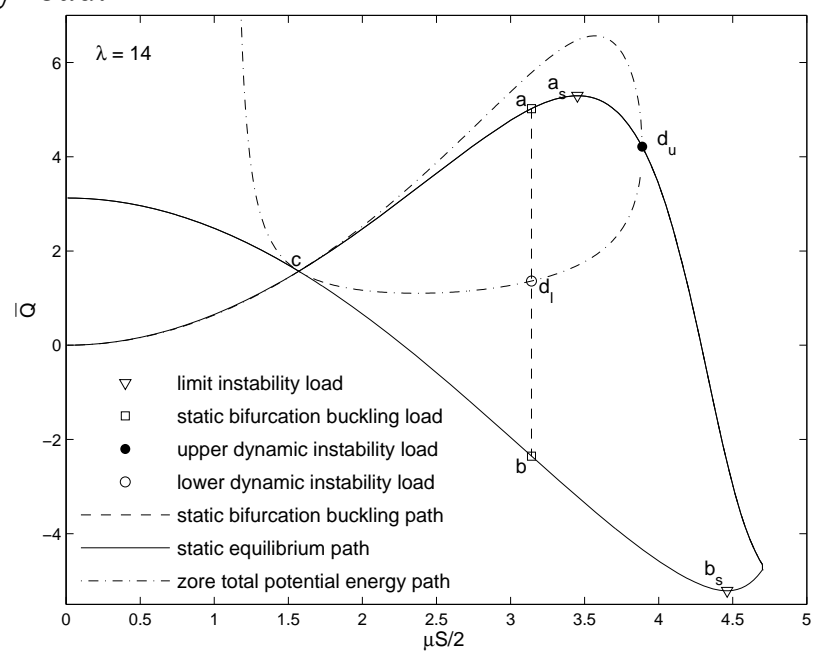

Figure 9. Upper and Lower Dynamic Instability Loads

The variations of the dimensionless upper and lower elasto-dynamic instability loads $Q S^{2} / 16 E I_{x}$ with the arch geometric parameter $\lambda$ are shown in Figure 10. It can be seen that the elasto-dynamic instability load increases with an increase of the arch geometric parameter. The variations of the elasto-static instability loads $Q S^{2} / 16 E I_{x}$ of the arches given by Eqs. 25, 29, and 36 with the geometric parameter $\lambda$ are also shown in Figure 10. It can be seen that both the upper and the lower elasto-dynamic instability loads are lower than the corresponding elasto-static instability load.

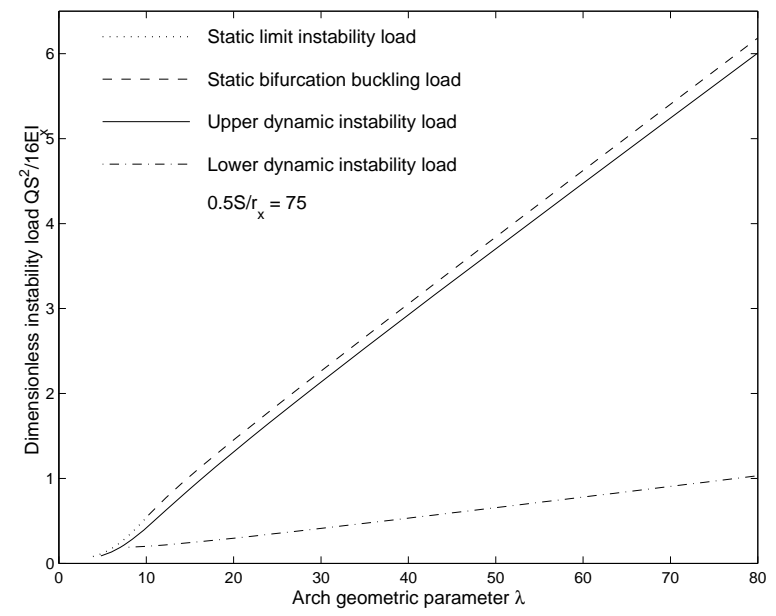

Figure 10. Comparisons of Dynamic Instability Loads with their Static Counterparts

\section{CONCLUSIONS}

The principle of conservation of energy was used in this paper in conjunction with a one degree-of-freedom idealized arch system to establish an energy criterion for the elasto-dynamic instability of a conservative structural system. The merit of the criterion is that it can determine the critical instability load without the need to solve the equations of motion of the system. The energy criterion was applied to the elasto-dynamic instability analysis of shallow pin-ended circular steel 
arches that are subjected to central step loading with infinite duration. The exact total potential energy, and the exact primary equilibrium and the secondary bifurcation equilibrium paths have been obtained, which are essential for the elasto-dynamic instability analysis based on the principle of energy conservation. Analytical solutions for the upper and lower elasto-dynamic instability loads of shallow pin-ended arches under the central step loading with infinite duration have been obtained. Because they are based on the exact total potential energy and equilibrium paths, the solutions for the elasto-dynamic instability loads are accurate. It has been found that the elasto-dynamic instability load for a pin-ended arch increases with an increase of the arch geometric parameter. It has also been found that the elasto-dynamic instability load of a shallow pin-ended arch due to the central step loading is lower than its elasto-static counterpart.

\section{ACKNOWLEDGEMENTS}

This work has been supported by the Australian Research Council through Discovery Projects, and an Australian Research Council Federation Fellowship, awarded to the second author.

\section{REFERENCES}

[1] Bradford, M.A., Uy, B. and Pi, Y.-L., "In-Plane Elastic Stability of Arches under a Central Concentrated Load", Journal of Engineering Mechanics, ASCE, 2002, Vol. 128, No. 7, pp. 710-719.

[2] Pi, Y.-L., Bradford, M.A. and Uy, B., "In-Plane Stability of Arches", International Journal of Solids and Structures, 2002, Vol. 39, No. 1, pp. 105-125.

[3] Pi, Y.-L., Bradford, M.A. and Tin-Loi, F., "Nonlinear In-Plane Buckling of Rotationally Restrained Shallow Arch under a Central Concentrated Load", International Journal of Non-Linear Mechanics, 2008, Vol. 43, No. 1, pp. 1-17.

[4] Pi, Y.-L., Bradford, M.A. and Tin-Loi, F., "Nonlinear Analysis and Buckling of Elastically Supported Circular Shallow Arches", International Journal of Solids and Structures, 2007, Vol. 44, No. 7-8, pp. 2401-2425.

[5] Pi, Y.-L. and Trahair, N.S., "Non-Linear Buckling and Postbuckling of Elastic Arches", Engineering Structures, 1998, Vol. 20, No. 7, pp. 571-579.

[6] Budiansky, B. and Roth, R.S., "Axisymmetric Dynamic Buckling of Clamped Shallow Spherical Shells", Collected Papers on Instability of Shell Structures, NASA TN D-1510, 1962.

[7] Budiansky, B. and Hutchinson, J.W., "Dynamic Buckling of Imperfection-Sensitive Structures", Proceedings XI International Congress of Applied Mechanics, Munich, 1964.

[8] Hsu, C.S., "Stability of Shallow Arches against Snap-Through under Timewise Step Loads", Journal of Applied Mechanics, 1968, Vol. 35, No. 1, pp. 1-39.

[9] Simitses, G.J., "Dynamic Stability of Suddenly Loaded Structures", Springer-Verlag, New York, USA, 1990.

[10] Lo, D.L.C. and Masur, E.F., "Dynamic Buckling of Shallow Arches", Journal of the Engineering Mechanics Division ASCE, 1976, Vol. 102, No. EM3, pp. 901-917.

[11] Gregory, W.E. Jr. and Plaut, R.H., "Dynamic Stability Boundaries for Shallow Arches", Journal of the Engineering Mechanics Division ASCE, 1982, Vol. 108, No. EM6, pp. 1036-1050.

[12] Donaldson, M.T. and Plaut, R.H., "Dynamic Stability Boundaries of a Sinusoidal Shallow Arch under Pulse Loads", AIAA Journal, 1983, Vol. 21, No. 3, pp. 469-471. 
[13] Matsunaga, H., "In-Plane Vibration and Stability of Shallow Circular Arches Subjected to Axial Forces", International Journal of Solids and Structures, 1996, Vol. 33, No. 4, pp. 469-482.

[14] Huang, C.S., Nieh, K.Y. and Yang, M.C., "In-Plane Free Vibration and Stability of Loaded and Shear Deformable Circular Arches", International Journal of Solids and Structures, 2003, Vol. 40, No. 22, pp. 5865-5886.

[15] Kounadis, A.N., Gantes, C.J. and Bolotin, V.V., "Dynamic Buckling Loads of Autonomous Potential System Based on the Geometry of the Energy Surface", International Journal of Engineering Science, 1999; Vol. 37, pp. 1611-1628.

[16] Kounadis, A.N., Gantes, C.J. and Raftoyiannis, I.G., "A Geometric Approach for Establishing Dynamic Buckling Load of Autonomous Potential N-Degree-of-Freedom Systems", International Journal of Non-Linear Mechanics, 2004; Vol. 39, pp. 1635-1646.

[17] Pi, Y.-L. and Bradford, M.A., "Dynamic buckling of shallow pin-ended arches under a sudden central concentrated load", Journal of Sound and Vibration, 2008, Vol. 17, No. 3-5, pp. 898-917. 